\title{
Different protocols for analyzing behavior and adaptability in obstacle crossing in Parkinson's disease
}

\author{
Nafiseh Mollaei' \\ Estela Bichol \\ Nuno Sousa ${ }^{2,3}$ \\ Miguel Fernandes Gago ${ }^{2-4}$ \\ 'Centro Algoritmi, Campus Azurem, \\ University of Minho, Guimarães, \\ ${ }^{2}$ Life and Health Sciences Research \\ Institute (ICVS), School of Medicine, \\ University of Minho, Braga, ${ }^{3}$ ICVS- \\ 3B's-PT Government Associate \\ Laboratory, Braga, ${ }^{4}$ Neurology \\ Department, Hospital da Senhora da \\ Oliveira, Guimarães, Portugal
}

This article was published in the following Dove Press journal:

Clinical Interventions in Aging

7 November 2017

Number of times this article has been viewed

\begin{abstract}
Imbalance and tripping over obstacles as a result of altered gait in older adults, especially in patients with Parkinson's disease (PD), are one of the most common causes of falls. During obstacle crossing, patients with PD modify their behavior in order to decrease the mechanical demands and enhance dynamic stability. Various descriptions of dynamic traits of gait that have been collected over longer periods, probably better synthesize the underlying structure and pattern of fluctuations in gait and can be more sensitive markers of aging or early neurological dysfunction and increased risk of falls. This confirmation challenges the clinimetric of different protocols and paradigms used for gait analysis up till now, in particular when analyzing obstacle crossing. The authors here present a critical review of current knowledge concerning the interplay between the cognition and gait in aging and PD, emphasizing the differences in gait behavior and adaptability while walking over different and challenging obstacle paradigms, and the implications of obstacle negotiation as a predictor of falls. Some evidence concerning the effectiveness of future rehabilitation protocols on reviving obstacle crossing behavior by trial and error relearning, taking advantage of dual-task paradigms, physical exercise, and virtual reality have been put forward in this article.
\end{abstract}

Keywords: gait, obstacle crossing, cognition, behavior, Parkinson's disease, aging, falls

\section{Introduction}

\section{Postural control}

Postural balance is a complex task performed by the central nervous system (CNS), which involves feedback integration of multiple sensory inputs, including those from the vestibular, auditory, visual, and motor systems, based on an internal body schema. ${ }^{1}$ Postural control is an essential pillar for independent gait, in coordination with locomotion generators, establishing, and maintaining the proper postural orientation of body parts relative to one another and within the limits of biomechanical constraints inherent to the person and to the task and environment, and also ensuring the dynamic stability of the moving body (Figure 1). ${ }^{2,3}$ According to Schoneburg et al, ${ }^{4}$ the dynamic postural control during locomotion can be characterized by the integration of the 4 main postural control domains: 1) balance during quiet stance; 2) reactive postural adjustments to external perturbations; 3 ) anticipatory postural adjustments (APAs) in preparation for voluntary movements; and 4) dynamic balance during the movements, such as gait. One of the most concerning features of several neurodegenerative diseases is postural instability, which increases the risk of falls. ${ }^{5}$ In fact, postural instability is one of the disabling symptoms of Parkinson's disease (PD), with falls occurring in 46\% people, 


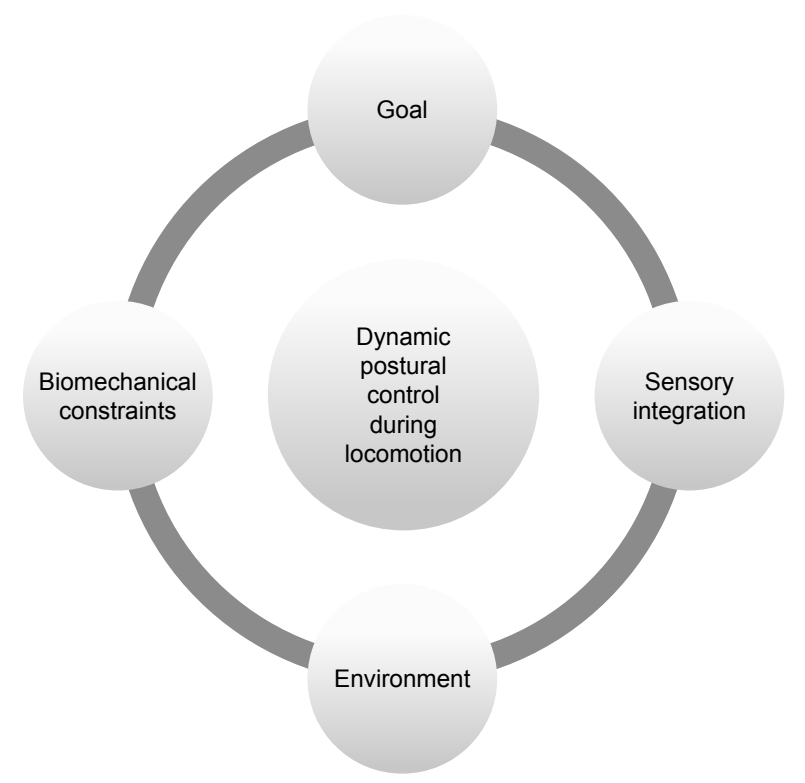

Figure I The model describing the elements that affect dynamic postural control during locomotion.

Note: Copyright (C) 2013. John Wiley and Sons. Reproduced from Earhart GM. Dynamic control of posture across locomotor tasks. Mov Disord. 2013;28(II): I50I-1508. ${ }^{3}$

in a given period of 3-months. ${ }^{6}$ As such, preserving gait is fundamental in PD, where independent gait is threatened by falls and multisystem deconditioning, thus requiring lifestyle and behavioral changes that warrant sustained and constant physical activity and exercise. ${ }^{7}$

\section{Obstacle crossing}

Crossing obstacles is a common challenge encountered in everyday life, where the human body has to adjust the visual perception as well as the length and height of each step to avoid contact in order to calculate how to pass the obstacle properly. The limitations in gait adaptability might be one of the reasons that put the elderly people at a greater risk of tripping and falling when crossing obstacles under timeconstrained conditions. ${ }^{8}$ A recent within-group comparison by Caetano et $\mathrm{al}^{8}$ has shown that young individuals maintain the same walking pattern for both the baseline and walkthrough conditions, whereas older adults reduce their step length, step velocity, and increase their double-support time significantly in walk-through conditions. As compared with their younger counterparts, the older adults demonstrate a more conservative adaptation strategy, exhibit poorer stepping accuracy, and make more stepping errors. As such, the authors concluded that reduced gait adaptability might place older adults at an increased risk of falling when accomplishing unexpected hazards. ${ }^{8}$ Stegemöller et $\mathrm{al}^{9}$ have also shown that PD patients change their behavior when crossing an obstacle, opting for a more conservative strategy, including a reduction in the mechanical demands and increase in dynamic stability during obstacle avoidance tasks. Galna et $\mathrm{al}^{10}$ have shown that PD patients inadvertently increase their step width when crossing an obstacle, as a compensation strategy, rather than increasing foot height as seen in healthy subjects. PD patients do not place their foot close enough to the front of the obstacle, as compensation for their reduced step length, being, therefore, more likely to step over on the obstacle while crossing it. In fact, the ability to make adjustments in gait to safely clear obstacles at home and in the community, like in rugs, shower recesses, and roadside curbs, is compromised in PD. ${ }^{10}$

\section{Risk of falling}

Elements attributed to an individual's risk of falling are commonly evaluated at 3 levels: 1) intrinsic factors, occurring at the level of the individual; 2) extrinsic factors, occurring at the level of the environment; and 3) activity-related factors. Falls are one of the most serious and common medical issues affecting the older adults. Indeed, many of the consequences of falls are psychological, including the fear of falls. There is a growing body of evidence that suggests the fear of falls may elicit a self-behavior and mind state that auto-perpetuates the state of risk of falling, by reducing physical and functional activity. ${ }^{11}$ Pan et al $^{12}$ investigated the mechanics of obstacle crossing in older adults that have been classified as having low or high risk of falling in accordance with having a score higher or inferior to the cut-off of 36 on the Tinetti Balance and Gait scale, respectively. The kinematics of the leading and trailing leg was evaluated when crossing an obstacle with different heights of $10 \%, 20 \%$, and $30 \%$ of leg length. Increasing the height of the obstacle led to increased toe-obstacle distance, and shortened swing phase of the leading limb, with a greater adaptation of clearance height of the trailing leg. Adults at a higher risk of falling exhibited a greater toe-obstacle clearance of the leading leg, the lesser symmetry between the leading and trailing legs, and a narrower step width. As such, according to this study, this gait behavior and obstacle crossing adaptability, may be associated with an increased likelihood of tripping and falling. ${ }^{12}$

\section{Motor and cognitive rehabilitation}

There is increasing evidence that motor rehabilitation can be employed to improve a safer obstacle-crossing behavior. In the last few years, there has been a growing body of scientific research where rehabilitation has been merged with new technologies. Caetano et $\mathrm{al}^{13}$ appraised the influence of home-based interactive video game step training on 
gait analysis performance in PD patients. Gait adaptability performance was evaluated at baseline and 12 weeks of follow-up, using different challenging obstacle paradigms (obstacle avoidance, short stepping target, long stepping target and no target/obstacle). According to this study, a home-based interactive step training was able to improve gait adaptability in response to unpredictable stepping targets in PD patients. Liao et $\mathrm{al}^{14}$ examined the effects of virtual reality-based exercise on obstacle-crossing performance and dynamic balance in individuals with PD. Compared with a traditional group, the virtual reality-based Wii Fit exercise, as part of a multifaceted training intervention was effective in improving obstacle-crossing performance, dynamic balance, functional ability, and the quality of life in PD patients. During the daily life challenges of crossing obstacles, besides the visual context and inputs coming from the environment, auditory inputs have also been shown to provide meaningful spatial cues or, in contrast, constitute contra-productive information. ${ }^{15,16}$ There is already substantial evidence that auditory cues, especially rhythmic auditory cues with action relevance (motor and sensorial relevance) (eg, wearing the sound of the steps in the gravel), improves gait in PD ${ }^{17}$ Still, there is lacking information concerning the effect of auditory cues during obstacle crossing and/or gait adaptability. Pestana et $\mathrm{a}^{18}$ investigated the effect of auditory cues on gait asymmetry in patients with PD compared with that in healthy subjects in an unobstructed environment and in an environment with an obstacle. It was found that auditory cues decreased gait asymmetry in PD in an unobstructed environment; they failed to provide any benefit during obstacle crossing, showing no significant effect on the asymmetry of gait in PD. The aforementioned studies support the concept that, in addition to muscle strengthening, the cognition and adaptability of behaviors of gait during obstacle crossing are also possible and clearly warrant further investigation. It can thus be further hypothesized that by trial and error learning mechanisms, even when neuropathology has disturbed spinal and supraspinal automatic neural network involved in postural control and locomotion, the crossing behavior can be revived and relearned in order to achieve a new and better outcome.

In the backdrop of the aforementioned context, the objectives of this paper are to provide a critical review concerning 1) different gait analysis measures and respective clinometric properties, regarding clinical progression and treatment outcomes; 2) the interplay between cognition and gait and how different apparatus and paradigms can be used to study different domains in gait and postural control;
3) and ultimately how obstacle-crossing behavior adaptability may be compromised in patients with $\mathrm{PD}$, putting forward potential translational physiotherapy opportunities.

\section{Clinimetric properties of gait analysis}

Three-dimensional kinematic measures of gait are used in clinical gait analysis, ${ }^{19}$ and still, continues to be the goldstandard critical measure for gait research and clinical practice. However, it requires highly specialized staff to run these systems; their high cost and long preparation time are hurdles to their wholesale adoption as quotidian clinical tools. In the last decade, body-worn sensors have been proven to be an alternative tool for motion analysis, with growing applications in movement disorders, particularly in the analysis of posture, balance, and gait in PD. ${ }^{20}$ The measurements of gait can be aggregated into 2 group domains, including 1) spatiotemporal characteristics that demonstrate a typical cycle, described as the mean of multiple steps; and 2) dynamic trait of gait that expresses the inconsistency of spatiotemporal measures across multiple steps. ${ }^{21}$ The spatiotemporal characteristics of gait are a reliable and valid measurement for healthy controls who are aging and also for inferring deviation from normality and diagnosing neurodegenerative diseases. ${ }^{22}$ Gait speed is the most commonly used spatiotemporal gait parameter owing to its simplicity and extensive historic backup research. It is primarily considered as the primary outcome in gait rehabilitation clinical studies. ${ }^{23}$ Yet, though gait speed may be the absolute reflection of the overall global gait performance, it does not reveal the fundamental debilitations (eg, reduced step length and step width) or the underlying pathology that is affecting the performance. ${ }^{24}$ Gait variability, reflecting the dynamic trait of gait, has been presented as an alternative variable, showing good correlation with the impairment of gait along with clinical progression and severity of the disease. ${ }^{21}$ Several descriptions of the dynamic traits of gait, collected over long periods, may unravel the structure and pattern of fluctuations in gait and underlying pathology. Certainly, gait variability and fractal scaling of gait break down with age and hence may be sensitive markers of early dysfunction in PD. ${ }^{25}$ In fact, both coordination and consistency can be combined into a single element of gait performance. ${ }^{3}$ However, in a systematic review concerning the therapeutic interventions and rehabilitation in aged adults and gait variability assessment, several critical issues have been highlighted; and a large number of variability outcome variables have already been published without a consensus for robust estimates. ${ }^{26}$ The discriminative and predictive validity of gait variability 
has been inferred, using principal components of factor statistical analysis, from cross-sectional and longitudinal studies rather than being formally examined. ${ }^{27} \mathrm{~A}$ study by Brach et al provided point estimates of meaningful change for swing time and stance time variability in 241 communitydwelling older adults based on 2 annual follow-up analyses, although CIs were not reported. ${ }^{28}$ The measurement of gait dynamics (gait variability) has already been proposed as an outcome measurement in pharmacological clinical trials, particularly in assessing the benefit of donepezil in reducing the risk of falls in older people with mild cognitive impairment. ${ }^{29}$ However, given the concerns regarding the reliability of different sensors and assessment of gait by different protocols and different environments, the assumption of the variability of one particular gait parameter as the ultimate and final answer is too early. Hence, continued development of robust and practical protocols and accessible processing techniques is paramount to help us understand the contribution of gait dynamics and the pretense of its use as an outcome measurement in clinical trials better.

\section{Interplay between cognition and gait}

Over the past decade, several studies have emphasized on the interplay between balance, gait, and risk of falls on the one hand, and cognitive function and risk of dementia on the other hand. ${ }^{30}$ Cognitive deficits deteriorate with aging and may even cause gait impairment, increasing the risk of falls, particularly during more challenging situations. Nearly $60 \%$ of elderly people with cognitive impairment fall every year, which is 2-fold more than that observed among their cognitively intact peers. Figure 2, extracted from a study by Amboni et al, ${ }^{31}$ properly depicts the coexistence of cognitive impairment and gait abnormalities, as well as dementia and increased risk of falls. Conversely, there is a growing body of data showing that gait abnormalities may predict the future development of dementia and that cognitive impairment increases the risk of falls. ${ }^{31}$ The executive function refers to a set of higher order cognitive processes that control, integrate, organize, and maintain other cognitive abilities. It appears that the dorsolateral prefrontal cortex, a structure of the brain that is associated with executive function and attention, is also associated with several gait domains, which further highlights the relationship between cognition and gait, and the effect of interventions enhancing executive function. ${ }^{32}$

Another group of researchers suggests that cognitive interventions may have a carry-over effect from cognitive to physical gait domains, thus providing a dual beneficial effect, reducing dual-task costs and the risk of falling. A study by

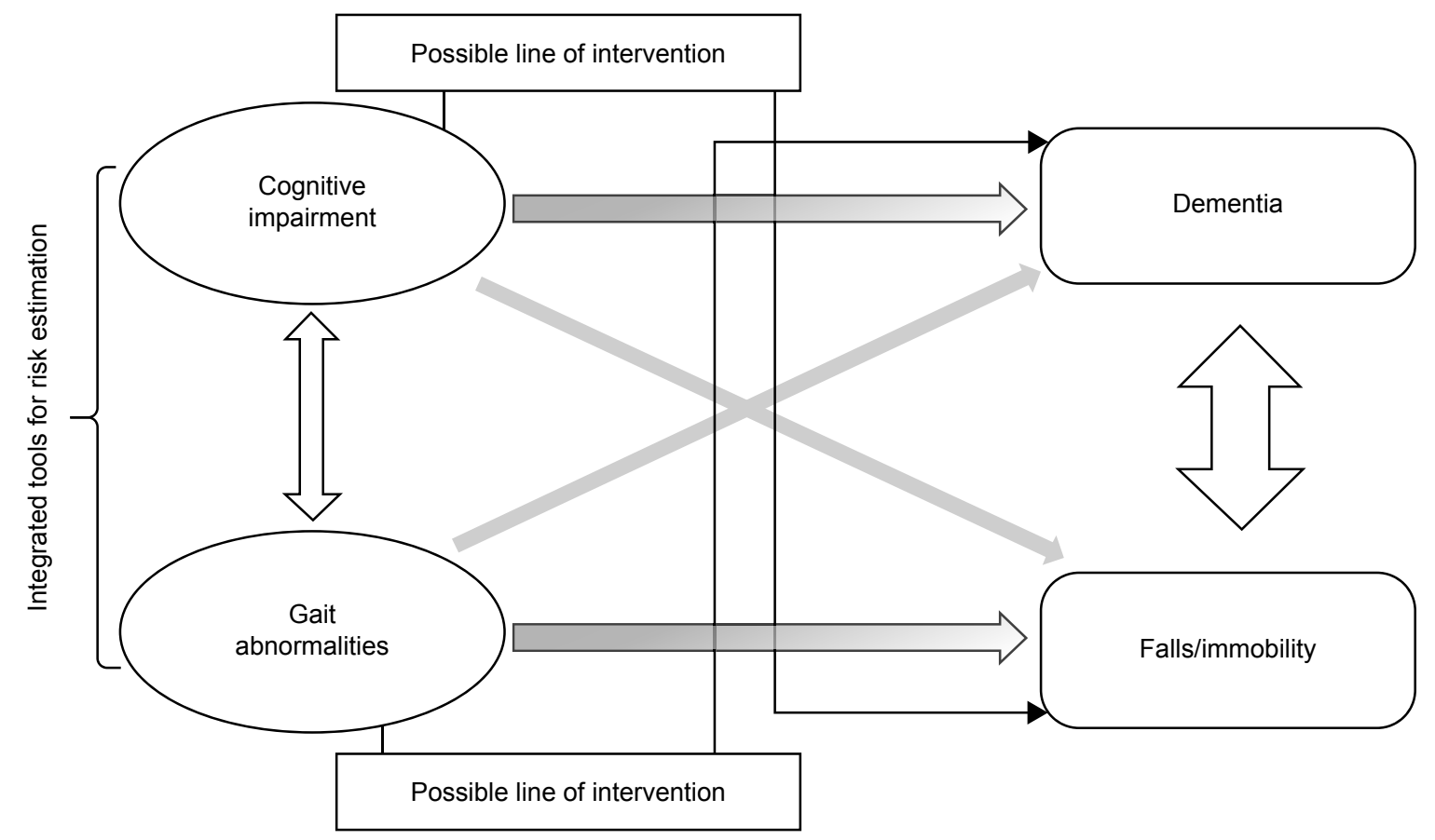

Figure 2 Cognitive impairment predicted dementia, although gait abnormalities increased the risk for falling (shaded arrows).

Notes: A complete understanding suggests that cognitive impairment and gait abnormalities, coupled with dementia and falls, are related to each other (white arrows). In the light of recent evidence, gait abnormalities predict dementia and cognitive impairment raises fall risk (gray arrows); based on the close relationship between cognition and gait on the one hand, integrated tools for risk estimation are needed (bracket). Furthermore, the possible lines of intervention (black arrows) could rely on increasing cognition for fall prevention and on walking training for lessening dementia risk. Copyright (C) 20I3. John Wiley and Sons. Reproduced from Amboni M, Barone P, Hausdorff JM. Cognitive contributions to gait and falls: evidence and implications. Mov Disord. 2013;28(I I): I520-1533. ${ }^{31}$ 
Verghese et al, ${ }^{33}$ involving sedentary seniors who played "computer games", showed an improvement in usual walking and dual-tasking gait speed, and this clearly illustrates the potential of cognitive therapy.

Cognition plays a dominant role in gait preservation while pathology and/or age dampens the automaticity of gait; however, the interpretation of the interplay between cognition and gait may be influenced by the selection of gait measures, protocols, and the analytic approach. ${ }^{34}$ In the following section, some of these issues have been considered.

\section{Different apparatus and paradigms to assess gait Normal walking}

The assessment of gait speed at self-selected pace over 4 meters is the most commonly used, as it is a quick and safe method, being clinically relevant for the evaluation of risk of falls, cognitive impairment, institutionalization, and mortality. ${ }^{35}$ A departure from this strategy is acceptable for some gait characteristics but not for all. Gait speed in PD has proven to be reliable, with good test-retest reliability, over 10 meters and 6 minutes for comfortable self-selected gait speed and for fast walking in clinical and home environments. ${ }^{36,37}$ However, this is not the case for gait variability, where reliability and clinimetrics improve with increasing number of steps, requiring at least 120 steps. $^{38,39}$ Gait is less variable when walking continuously, like on a treadmill, compared with that when performing multiple short intermittent walks. ${ }^{40}$ This clearly highlights the importance of the testing protocol, which is how different apparatus and paradigms influence the outcomes. In a study by Galna et al, ${ }^{38}$ gait variability was measured twice with an interval of 1 week in 27 older adults and 25 PD patients. The participants walked at their preferred pace during 1) a continuous 2-minute walk and 2) 3 intermittent walks over a 12-m walkway. The gait variability was calculated as the within-person standard deviation for step velocity, length and width, and step, stance and swing duration. The authors found that continuous walking protocols may be more reliable as they measure gait under steady state conditions thus reflecting more automatic gait control unconfounded by the repeated shifts of attention associated with short intermittent walks (Figure 3).

\section{Treadmill}

Another important question is whether the evaluation in treadmills is a good protocol to infer the underlying gait pathology. Even more, it is even questionable whether the treadmill assessment appropriately reflects the normal ground walking. Analyzing gait in a treadmill is a considerably more practical system of analyzing gait in a clinical environment but, more importantly, it may form the last resort when normal walking on flat ground is not feasible in advanced stages of neurological diseases. Moreover, in patients with considerable postural instability only being able to walk with external help, a treadmill with a harness may be the only system to adequately measure rehabilitation protocols. ${ }^{41}$ Indeed, it has been demonstrated that treadmill training improves gait speed, stride length, without improving cadence or walking distance. ${ }^{41}$ The comparison of 2 walking training programs, one performed on a treadmill and another over the ground, showed that only the former led to improvement in the stride length at the preferred and maximal speed in PD. ${ }^{42}$ These findings justify the use of treadmill as a potential therapeutic approach in PD patients. However, treadmill limits the assessment of some gait variables, such as gait variability, probably due to the proprioceptive cues and rhythmicity provided by the belts of the treadmill.

\section{Dual-task paradigms}

In contrast to single paradigms (eg, walking free at desired running speed) in dual-task paradigms, the patient also

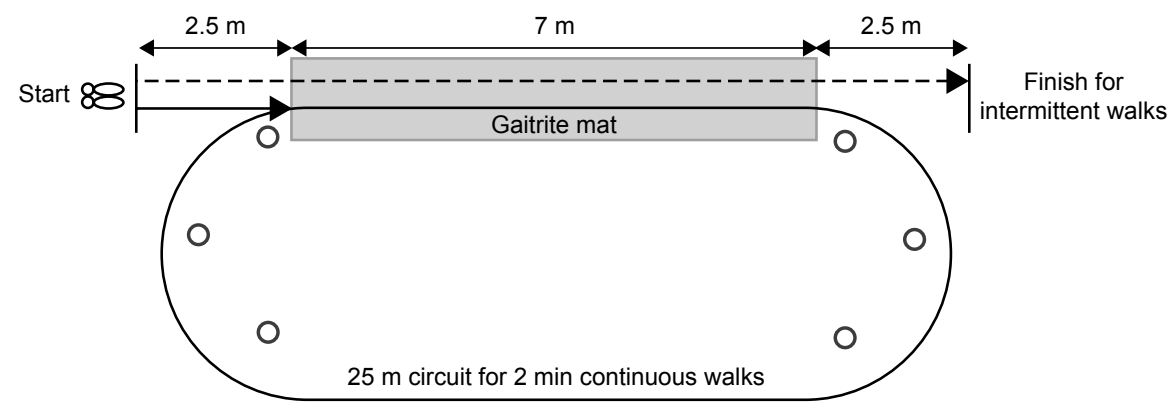

Figure 3 This figure depicts the laboratory setup for testing continuous and intermittent walks.

Notes: The authors highlight that continuous walking protocols may be more reliable. Reprinted from Gait Posture, 37(4), Galna B, Lord S, Rochester L, Is gait variability reliable in older adults and Parkinson's disease? Towards an optimal testing protocol, 580-585, Copyright (20I3), with permission from Elsevier. ${ }^{38}$ 
performs a cognitive task (eg, walking and arithmetic) on top of the automatic locomotor and postural control. Dual tasks are mainly cognitive, and they vary widely from a simple "walking while talking" to counting backward in serial order. Some of these cognitive tasks may be concurrent with the same networks that are also involved in postural and locomotor control. ${ }^{31}$ This evidence has reinforced the use of dual-tasking paradigms in order to decrease compensatory cognitive control on gait and make latent motor deficits more emerging. Consequently, dual-tasking paradigms also expose the level of compensatory cognitive control required to maintain gait performance. Indeed, walking under cognitive strain; walking while talking, by cognitively stressing locomotion demands, might help in the identification of dysfunction much early, and are therefore a more sensitive measure of the underlying pathology, and a robust predictor of frailty, disability, and mortality in high-functioning older adults. ${ }^{43}$ In PD, Stegemoller et $\mathrm{al}^{44}$ have shown that impaired gait performance under dual-tasking conditions (eg, slower gait speed and increased variability) is intrinsic to PD and correlated to the cognitive processing speed. However, caution is required while using these protocols, because dual-task interference is also a feature of healthy aging. Recent work by Smulders et al, ${ }^{45}$ in a large cohort of PD patients, after correcting for baseline group differences, have questioned the advantages of dual-task paradigms for predicting falls due to the wide variation in response between different individuals. Pieruccini-Faria et $\mathrm{al}^{46}$ examined, under cognitively demanding conditions (with and without an auditory digit monitoring dual task), the role of dopaminergic treatment on gait when approaching an obstacle to be stepped over. They also assessed visual strategies in these conditions, as a reflection of movement planning. They concluded that dopamine influences planning by limiting sensorimotor processing capacity, especially in the presence of increased cognitive demand in PD. More interestingly, the factor that can be interpreted as a burden for gait performance can be potentially used as a tool for rehabilitation. Orit Segev-Jacubovski et al illustrated the potential utility of multiple, diverse forms of cognitive therapy for reducing the risk of falls. ${ }^{30}$ To sum up, although dual-task tests have been generally adopted, there is no consensus with respect to its optimal protocol, warranting further refinement in future studies.

\section{Obstacle crossing and negotiation behavior}

Balance impairments throughout walking and tripping when negotiating obstacles are the 2 most common causes of falls in older adults. ${ }^{12}$ Walking over obstacles implies 4 main tasks: 1) to maintain balance; 2) to prevent tripping during crossing; 3 ) to prevent slipping during landing; and 4) to progress the body forward. Inaccuracy in movement during obstacle crossing could result in tripping on the obstacle or slipping due to foot strike. In addition, a loss of balance while maneuvering an obstacle may result in sudden and inappropriate movements of the lower limbs that lead the foot to contact the obstacle rather than ignore it. Pan et $\mathrm{al}^{12}$ have shown, in older adults, that the height of the obstacle increases the strategy of the leading limb changes. Moreover, the subjects with increased risk of falls demonstrated lesser symmetry between the leading and trailing legs and a narrower step width, which are the features that increase the likelihood of tripping. Caetano et $\mathrm{al}^{8}$ have shown that older adults shorten the previous step before obstacle crossing, reduce the velocity of both previous and obstacle steps and increase the time spent in double support. Also, the reduced step length, adopted by the older adults, correlated with the increased number of steps taken to approach the target. ${ }^{8}$

In $\mathrm{PD}$, there is mounting evidence that gait impairment is amplified during obstacle crossing. PD patients choose a more conservative strategy when crossing an obstacle, with a greater concern for safety margins in relation to the obstacle, leading to greater caution and slower speeds and increased toe clearance. ${ }^{9}$ This was proved in a study by Stegemöller et al, ${ }^{9}$ where the authors analyzed obstaclecrossing behaviors using a paradigm of 5 trials of normal walking and 5 trials of obstacle crossing. Regarding both tasks, the participants walked barefoot along a 9-m walkway at their normal, comfortable pace. The participants crossed the obstacle located in the middle of the path and continued walking to the end of the walkway. This lightweight obstacle was constructed of a wooden dowel crossbar $(91 \mathrm{~cm}$ long and $1.27 \mathrm{~cm}$ in diameter) and was supported by 2 vertical standards (Figure 4A). Regarding the obstacle clearance parameters, PD patients performed with a greater mean of vertical clearance height in both the leading and trailing feet traveling over the obstacle. In addition, PD patients, due to slower crossing speed, spent a longer time in a single support, positioned their stance foot closer to the object, and the leading foot helped them clear the object with a greater safety margin than their age-matched control subjects (Figure 4B).

Regarding the untouched issue, how the CNS controls postural stability during gait initiation with the goal to clear an obstacle, Yiou et $\mathrm{al}^{47}$ requested the healthy subjects to initiate gait as fast as possible in 3 different conditions of obstacle 
A

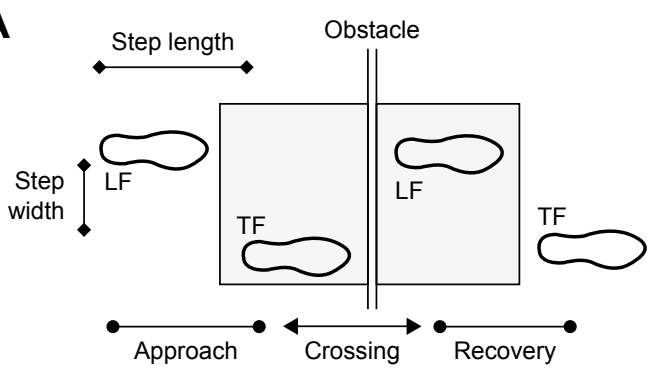

B
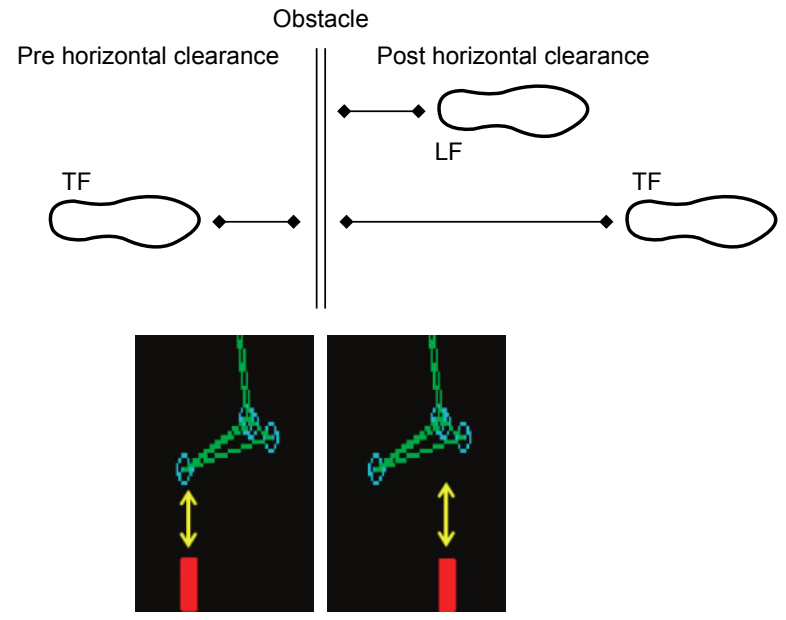

Vertical clearance

Figure 4 (A) Examples of apparatus and gait parameters used to calculate the approach, crossing, and recovery steps during obstacle crossing. (B) An example of horizontal and vertical obstacle clearance parameters.

Note: Reprinted from Arch Phys Med Rehabil, 93(4), Stegemöller EL, Buckley TA, Pitsikoulis C, Barthelemy E, Roemmich R, Hass C], Postural instability and gait impairment during obstacle crossing in Parkinson's disease, 703-709, Copyright (20I2), with permission from Elsevier. ${ }^{\text {? }}$

Abbreviations: LF, leading foot; TF, trailing foot. height, 3 conditions of obstacle distance, and 1 obstacle-free (control) condition. The authors investigated the stabilizing features of gait initiation (Figure 5), analyzing APAs, and lateral swing foot placement. In their work, it was found that the anticipatory peak of backward center-of-pressure shifted and the initial forward center-of-mass set decreased with obstacle height, probably in an order to limit the risk to trip over the obstacle, while the forward center-of-mass velocity at foot-off increased with obstacle distance, allowing a further step to be taken. Accordingly, the authors suggest that the CNS has the ability to predict the potential instability elicited by the obstacle clearance and that it scales the spatiotemporal parameters of APAs accordingly.

In PD, Rogers et a $1^{48}$ used a sudden drop or elevation of the support surface to assess the contribution of somatosensory information during gait initiation, comparing APAs for lateral weight transfer and propulsion. The authors have shown that PD patients have a longer APA duration, longer time to first step onset, and slower step speed than controls. This article suggests that a feed-forward neural control of gait initiation, regarding limb load and/or foot pressure, can modulate temporal and spatial components of step initiation. In this perspective, the coupling between posture and locomotion should be augmented in PD..$^{48}$

\section{Obstacle crossing behavior adaptability}

Interestingly, APAs can be increased to normal levels when a step is initiated in response to an external cue or when on

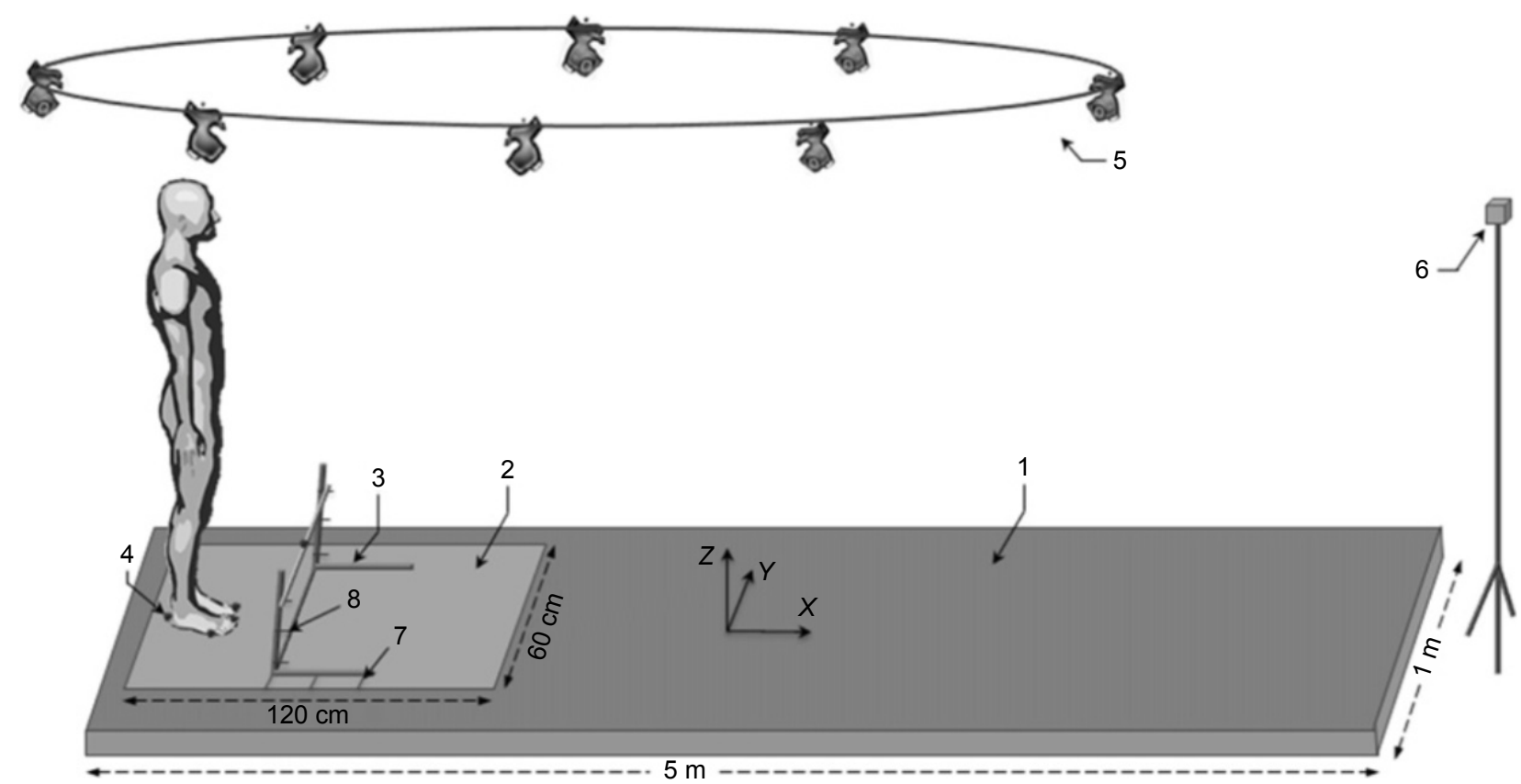

Figure 5 Schematic illustration of the experimental set-up analyzing anticipatory postural adjustments upon gait initiation crossing an obstacle.

Note: Reproduced from Yiou E, Artico R, Teyssedre CA, Labaune O, Fourcade P. Anticipatory postural control of stability during gait initiation over obstacles of different height and distance made under reaction-time and self-initiated instructions. Front Hum Neurosci. 2016;10:449.47 
levodopa. ${ }^{49}$ Without levodopa, the step initiation in PD subjects was characterized by decreased production of force, decreased the velocity of movement, and slowed execution of the APAs for self-generated step. These impairments were significantly less evident when the PD subjects were on levodopa therapy. Interestingly, an external stimulus improved and increased the force and the velocity of step initiation in healthy subjects and also in PD patients. In other words, dopaminergic therapy and an external stimulus similarly improved the deficient force production for the APAs associated with step initiation in PD. ${ }^{49}$

There is growing research on neurophysiology supporting rehabilitation interventions, focusing separately on isolated components of posture, balance, and gait in $\mathrm{PD} .{ }^{2}$ In addition, the rehabilitative interventions to assist lateral displacement of the APA may be beneficial in improving steps in patients with PD. Still, it is important to bear in mind that the coupling between posture and locomotion affects the effectiveness of these rehabilitation strategies, justifying a global approach. ${ }^{2}$ The ability to quickly adapt postural responses based on the task and environmental context is altered in PD, involves basal ganglia networks but probably is levodopa-resistant. ${ }^{50}$ Chong et $\mathrm{al}^{50}$ have shown that if the postural perturbation direction is altered unexpectedly, healthy adults immediately adapt their postural responses by changing postural muscle activation patterns; and, in contrast, PD patients take several trials before exhibiting the appropriate postural response to the new perturbations, unresponsive to levodopa medication. As such, the authors concluded that PD patients have more difficulty in using cognitive set to modify their responses, particularly when instructed to "resist" the perturbations. This may represent a relative "inflexibility" to alter postural responses to match changes in task conditions or contexts, or in other words, an impaired learning and adaptability, by trial and error, in PD. ${ }^{50}$ Still, in the work by Jacobs et al, ${ }^{51}$ it was shown that PD patients could increase their size of stepping responses to external perturbations when these patients could see their legs as they stepped toward a visual target. PD patients exhibited short compensatory steps due to abnormal proprioceptive-motor integration and used visual input to take longer compensatory steps when a target was provided, moderately responsive to levodopa in moderate stage PD patients. Still, in more severely impaired PD patients, neither visual input (viewing the target and in unobstructed vision) nor levodopa provided a significant improvement. These results led the authors to conclude that the degeneration of dopamine circuits within the basal ganglia is not responsible for the proprioceptive-motor deficit that degrades compensatory steps in PD. ${ }^{51}$ Interestingly, this innovative experiment paves alternative rehabilitation strategies using augmented proprioception, especially vision, in PD.

Chang et $\mathrm{l}^{52}$ determined the effect of Tai Chi Chuan exercises on obstacle-crossing behavior. In the study, 15 healthy older adults practicing Tai Chi Chuan (3 days a week for 5 or more years) (the Tai Chi group [TCG]) were compared with 15 normal healthy older adults who did leisure exercises (walking or jogging) for the same time. Kinematic parameters (by a Vicon motion analysis system) and ground reaction forces (by Kistler force plates) were synchronously recorded (Figure 6). The different crossing strategies between the TCG and the general group were addressed and it was found that the differences between the strategies accounted for the differences in obstacle-crossing performance in older adults. With regular Tai Chi Chuan training, older adults improved their physical capabilities and adopted a more efficient strategy, that is, paying less attention to crossing the obstacle and by increasing the vertical clearance of the leading foot and the vertical clearance of the trailing foot (defined as the minimum distances when the toe markers were vertically above the obstacle) by flexing the hip of the leading leg more, which enabled them to cross the obstacle safely and at a faster speed. The authors concluded that regular Tai Chi Chuan training in older adults may improve their physical capabilities and help in adopting a more efficient strategy. ${ }^{52}$

Table 1 summarizes the findings on the relationship between gait and cognition throughout obstacle crossing with aging, in young and old healthy subjects and in PD. In the aforementioned studies, exercise and/or physiotherapy

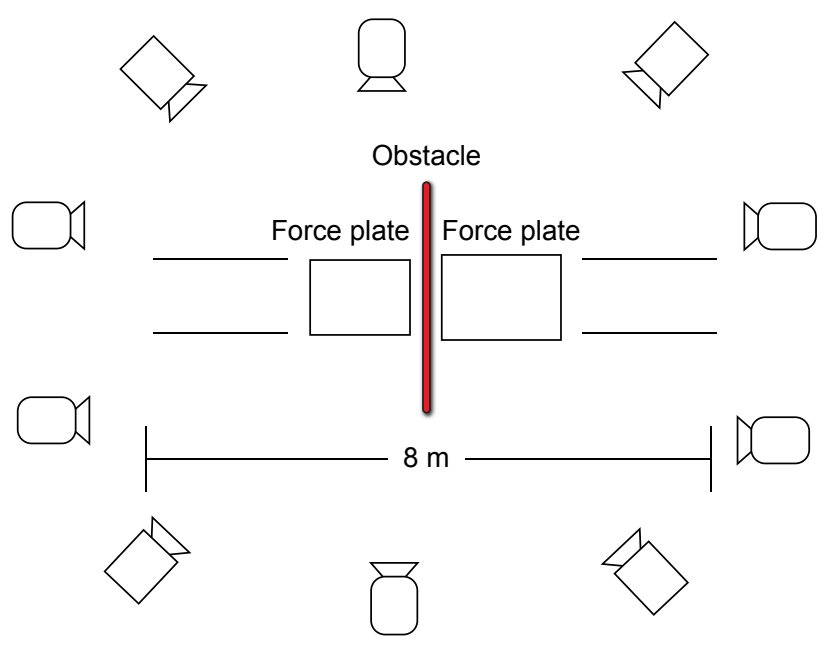

Figure 6 Top view of the laboratory setting of the effect of Tai Chi Chuan training in obstacle crossing behavior, analyzed by kinematics (Vicon motion analysis system) and kinetic ground reaction forces (Kistler force plates).

Note: The effect of Tai Chi Chuan on obstacle crossing strategy in older adults. Chang YT, Huang CF, Chang JH. Research in Sports Medicine. 26 Jun 20I5. Reprinted by permission of the publisher (Taylor \& Francis Ltd, http://www.tandfonline.com). ${ }^{52}$ 


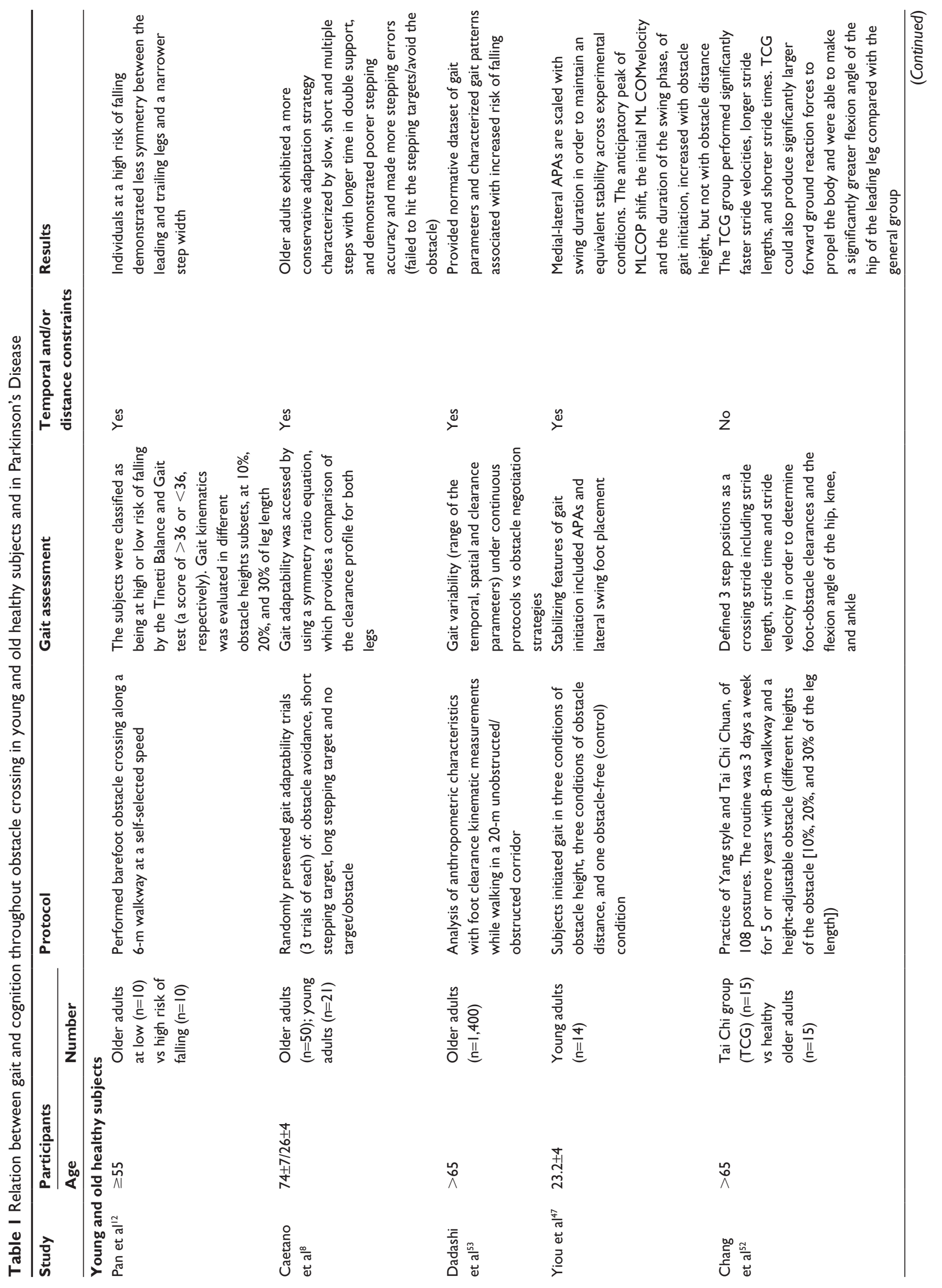




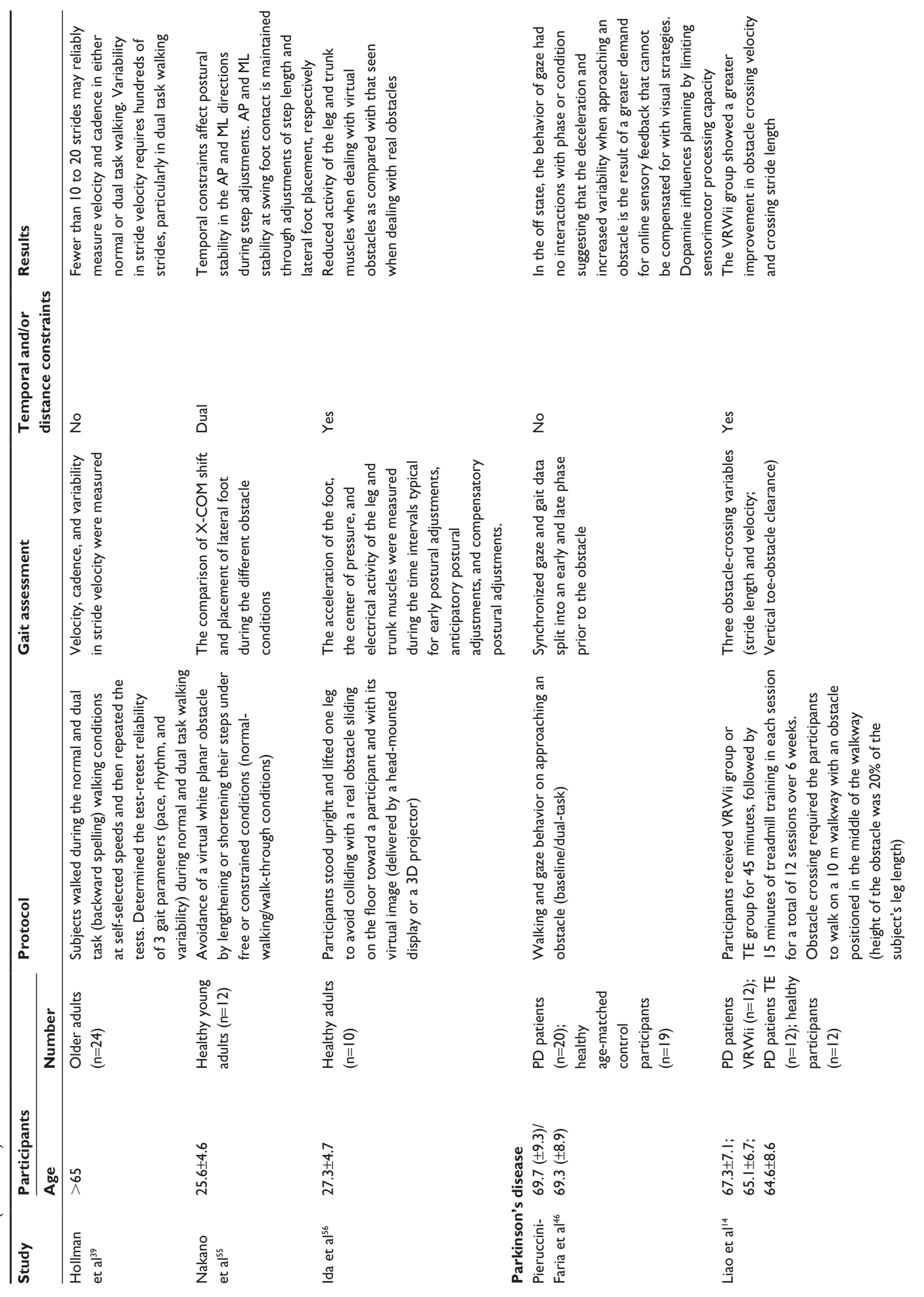



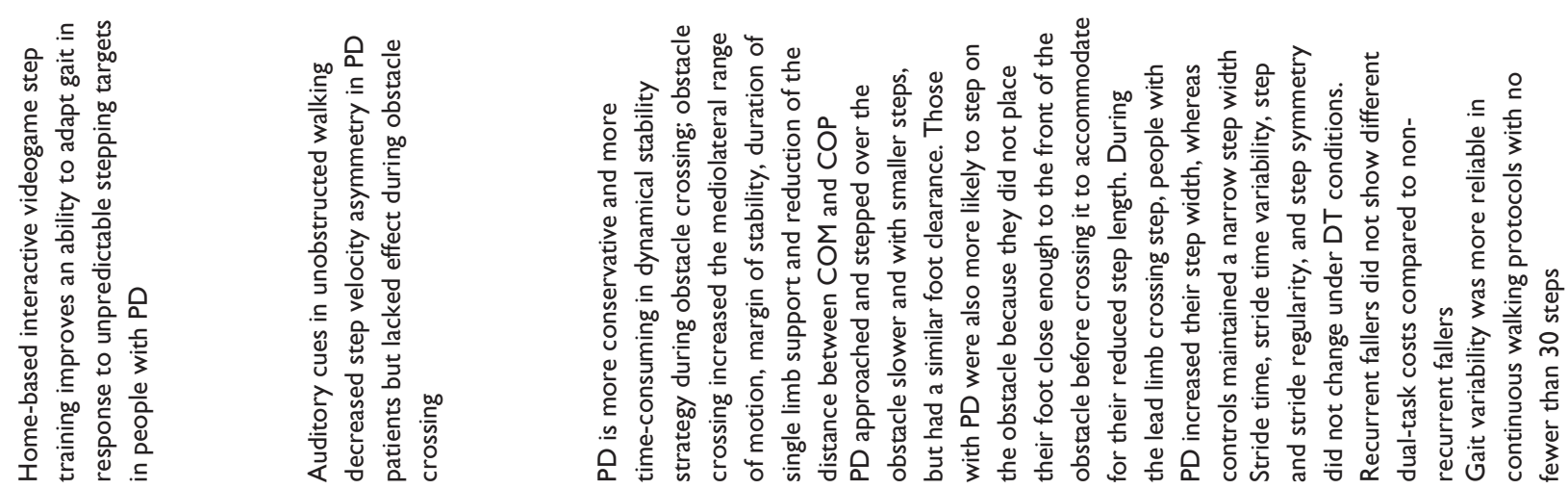

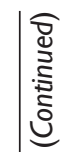
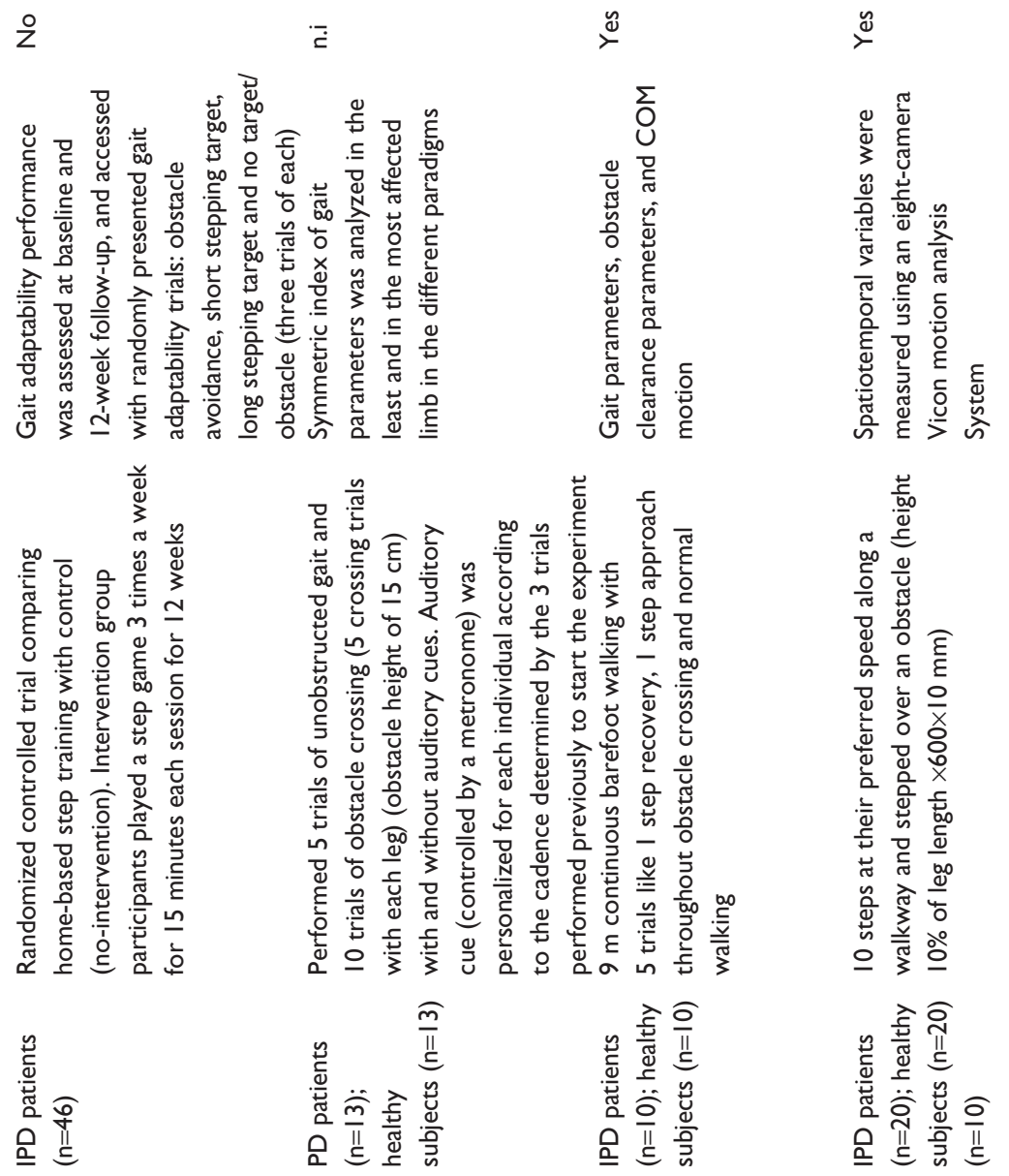

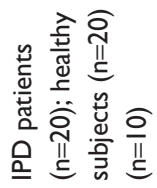
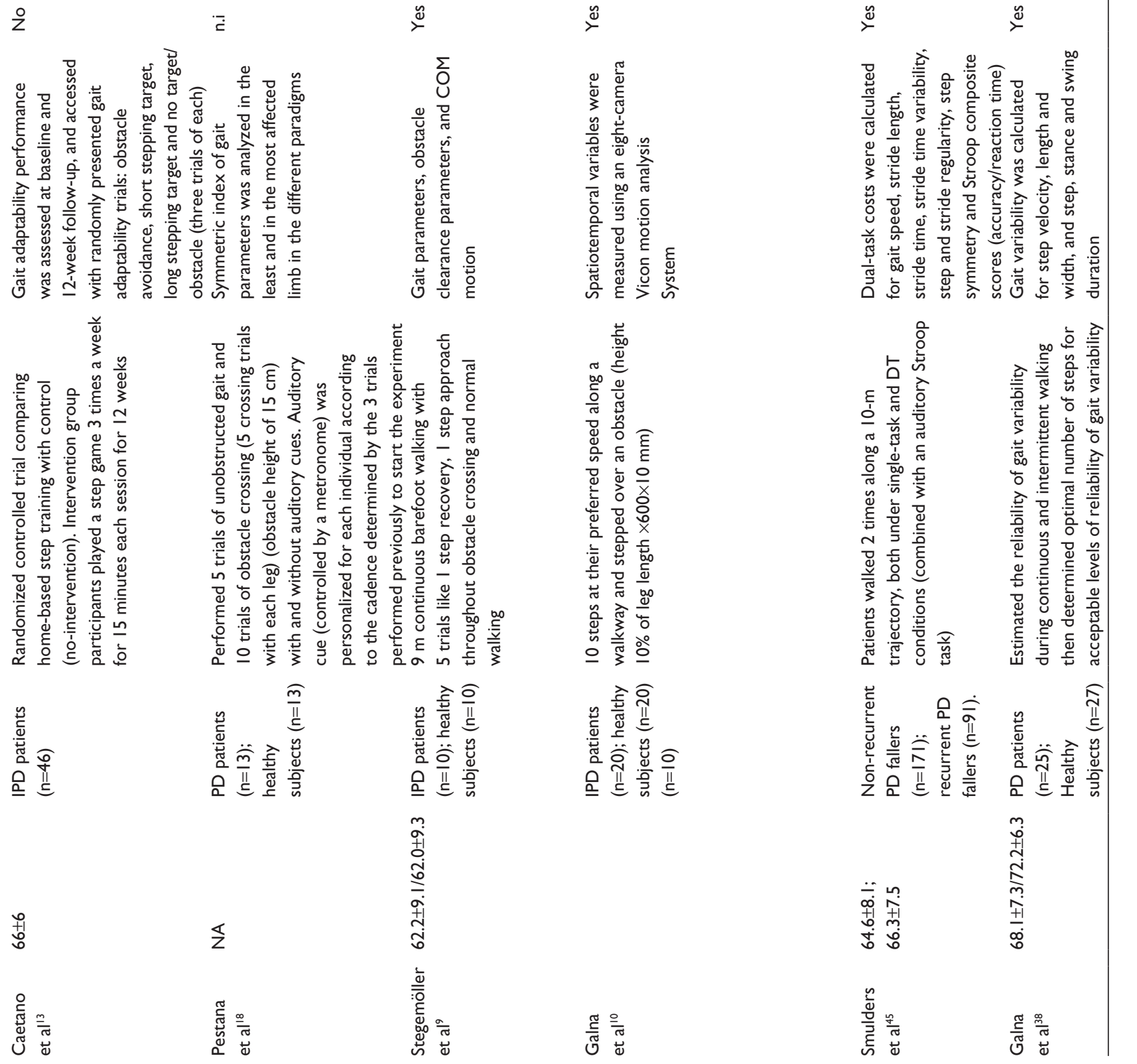


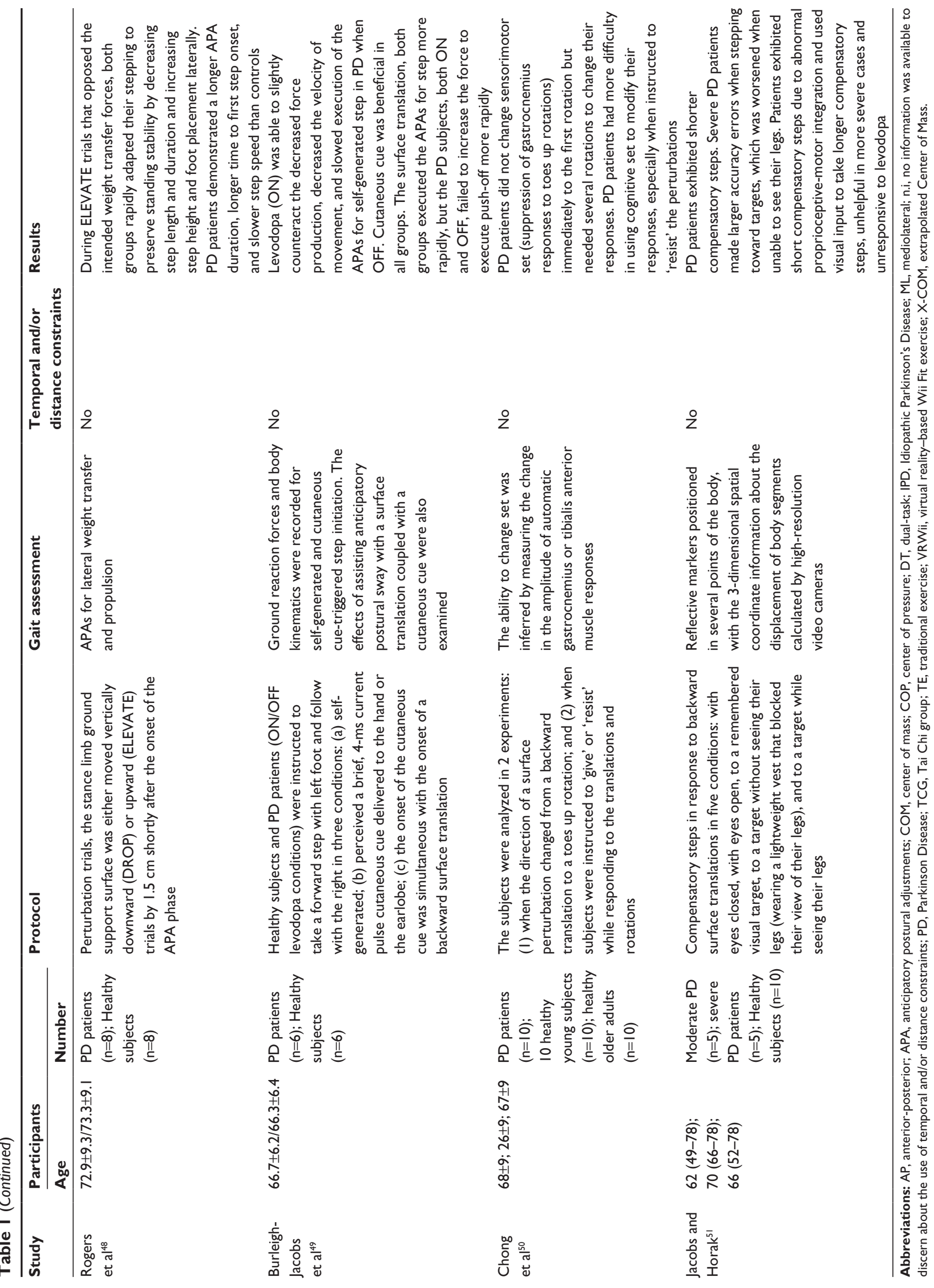


modulated and improved obstacle-crossing behavior and efficiency, not only in healthy older subjects but also in PD patients. It could, however, still be argued if these benefits are only due to an overall better muscular fitness or is a result of a combination of muscular and cognitive benefits, with the latter being consequent to the adaptability and relearning of crossing obstacle behaviors.

\section{Conclusions and future directions}

Despite the abundant literature on obstacle crossing in humans, the question of how the CNS controls postural stability and locomotion during obstacle crossing, remains unclear, even more in PD. The effect of aging on the ability to safely negotiate obstacles is paramount since difficulty in negotiating obstacles is one of the key determinants that lead older adults to refrain from walking. With regard to $\mathrm{PD}$, there is paramount evidence that PD patients present an increased risk of falls, such as during obstacle crossing. This increased risk of falling while obstacle crossing is not only due to impairment in postural control and locomotion but also because of an impairment in the gait adaptability and error trial relearning that involves cognitive behavior in PD. In this review article, the authors have put forward the current knowledge on the gait analysis, in particular, the clinimetric properties of obstacle-crossing behaviors during aging and in PD. Dadashi et $\mathrm{al}^{53}$ made a substantial work, on a large cohort of 1,400 healthy participants, establishing normative values for foot clearance and gait speed parameters. This is a crucial work in order to understand the complex relationship between gait and falls as well as obstacle-negotiation strategies, paving the way for future studies with different apparatus and paradigms. However, in contrast to spatiotemporal parameters (such as gait speed), the variability of gait may better reflect the dynamics of gait, bringing some order to chaos. ${ }^{21}$ In addition, when designing apparatus in laboratory-hospital environments, especially when seeking to analyze variability, hundreds of strides, particularly in dual-task conditions, should be collected for reliable data. ${ }^{39}$ In spite of all this, when setting apart from laboratory to ambulatory evaluation in future studies, this limitation will be over-ridden and other challenges, such as management of bigger data, will certainly arise.

The interplay between cognition and gait, ${ }^{30}$ and the influence of postural instability in PD in reducing the automaticity of gait putting a higher burden on cognitive compensatory strategies have also been discussed. This assertion has been put forward by taking advantage of the dual-task paradigms during gait analysis. However, thinking about the complexity of daily life environments, the results extracted in laboratory settings may be influenced by the fixed protocols, sometimes poorly ecological, and the analytic approach itself. ${ }^{34}$ Neurological diseases impair the structure and function of locomotor and postural control networks, and pathological and/or compensatory changes are reflected in slowness, increased variability, worst postural control and increased risk of falling. This is particularly evident in PD where postural instability is a critical landmark in the progression of the disease. In the present review, the authors have presented several arguments for considering obstacle crossing as a complex task, where several networks, postural control (eg, APAs), gait and cognition (eg, executive functions), visual perception (eg, environment constrictions; obstacle height) and multisensory integration (eg, proprioception), have all converged in order to avoid tripping and falling. In this context, gait analysis protocols taking the use of crossing obstacle paradigms can provide a quick screening tool to identify patients at a risk of falling and evaluate outcomes of training rehabilitation programs. In fact, several promising physiotherapy interventions have already shown robust scientific evidence, ${ }^{54}$ contemplating itself as a field of growing interest and investigation. The sensory information, regarding limb load and/or foot pressure, can modulate temporal and spatial components of step initiation and obstacle crossing, and in this perspective, augmenting the interplay between postural control and locomotion (eg, APAs) should be envisioned in future rehabilitation protocols. ${ }^{48}$

Once some of the aforementioned doubts are overridden, a better understanding of the impairments of obstacle crossing with aging and in neurological diseases such as PD will be achieved. This will further pave a way to envision the rehabilitation strategies focusing on the repetition and potentiating the learning effect. In addition to muscle strengthening, there is paramount evidence that by trial and error, the behavior of obstacle crossing can be revived and relearned in order to achieve a new and better outcome. Despite potentially slow rates of adaptation and learning in $\mathrm{PD}$, the so-called postural inflexibility of $\mathrm{PD},{ }^{50}$ patients can eventually relearn and adapt gait and crossing behavior with repetition. Further, the more breathtaking fact is the expectation that there is a carrying-over effect from cognitive to physical gait domains, thus providing a dual beneficial effect, in cognition, reducing dual-task costs, and risk of falling when crossing an obstacle. ${ }^{33}$

\section{Acknowledgment}

This work was partially supported by the projects NORTE01-0145-FEDER-000026 (DeM-Deus Ex Machina) 
financed by the Regional Operational Program of the North (NORTE2020) PORTUGAL2020 and FEDER, and FP7 Marie Curie ITN Neural Engineering Transformative Technologies (NETT) project.

\section{Disclosure}

The authors report no conflicts of interest in this work.

\section{References}

1. Peterka RJ, Loughlin PJ. Dynamic regulation of sensorimotor integration in human postural control. J Neurophysiol. 2004;91(1):410-423.

2. Mille ML, Creath RA, Prettyman MG, et al. Posture and locomotion coupling: a target for rehabilitation interventions in persons with Parkinson's disease. Parkinsons Dis. 2012;2012:754186.

3. Earhart GM. Dynamic control of posture across locomotor tasks. Mov Disord. 2013;28(11):1501-1508.

4. Schoneburg B, Mancini M, Horak F, Nutt JG. Framework for understanding balance dysfunction in Parkinson's disease. Mov Disord. 2013; 28(11):1474-1482.

5. Balash Y, Peretz C, Leibovich G, Herman T, Hausdorff JM, Giladi N. Falls in outpatients with Parkinson's disease: frequency, impact and identifying factors. $J$ Neurol. 2005;252(11):1310-1315.

6. Pickering RM, Grimbergen YA, Rigney U, et al. A meta-analysis of six prospective studies of falling in Parkinson's disease. Mov Disord. 2007; 22(13): 1892-1900.

7. Speelman AD, van de Warrenburg BP, van Nimwegen M, Petzinger GM, Munneke M, Bloem BR. How might physical activity benefit patients with Parkinson disease? Nat Rev Neurol. 2011;7(9):528-534.

8. Caetano MJ, Lord SR, Schoene D, Pelicioni PH, Sturnieks DL, Menant JC. Age-related changes in gait adaptability in response to unpredictable obstacles and stepping targets. Gait Posture. 2016;46: $35-41$.

9. Stegemöller EL, Buckley TA, Pitsikoulis C, Barthelemy E, Roemmich R, Hass CJ. Postural instability and gait impairment during obstacle crossing in Parkinson's disease. Arch Phys Med Rehabil. 2012;93(4):703-709.

10. Galna B, Murphy AT, Morris ME. Obstacle crossing in people with Parkinson's disease: foot clearance and spatiotemporal deficits. Hum Mov Sci. 2010;29(5):843-852.

11. Dionyssiotis Y. Analyzing the problem of falls among older people. Int J Gen Med. 2012;5:805-813.

12. Pan HF, Hsu HC, Chang WN, Renn JH, Wu HW. Strategies for obstacle crossing in older adults with high and low risk of falling. $J$ Phys Ther Sci. 2016;28(5):1614-1620.

13. Caetano MJD, Menant JC, Canning CG, et al. Effects of videogame step training on gait adaptability in people with Parkinson's disease - A randomized controlled trial [abstract]. Mov Disord. 2016;31 (Suppl 2).

14. Liao YY, Yang YR, Cheng SJ, Wu YR, Fuh JL, Wang RY. Virtual reality-based training to improve obstacle-crossing performance and dynamic balance in patients with Parkinson's disease. Neurorehabil Neural Repair. 2015;29(7):658-667.

15. Gago MF, Fernandes V, Ferreira J, et al. Role of the Visual and Auditory Systems in Postural Stability in Alzheimer's Disease. J Alzheimers Dis. 2015;46(2):441-449.

16. Gandemer L, Parseihian G, Kronland-Martinet R, Bourdin C. Spatial cues provided by sound improve postural stabilization: evidence of a spatial auditory map? Front Neurosci. 2017;11:357.

17. Young WR, Shreve L, Quinn EJ, Craig C, Bronte-Stewart H. Auditory cueing in Parkinson's patients with freezing of gait. What matters most: action-relevance or cue-continuity? Neuropsychologia. 2016;87:54-62.

18. Pestana MB, Gobbi LTB, Simieli L, et al. Auditory cues during obstacle crossing increase gait asymmetry in neurologically health individuals but not in people with Parkinson's disease [abstract]. Mov Disord. 2016;31(Suppl 2).
19. Jansson G, Johansson G. Visual perception of bending motion. Perception. 1973;2(3):321-326.

20. Bloem BR, Marinus J, Almeida Q, et al; Movement Disorders Society Rating Scales Committee. Measurement instruments to assess posture, gait, and balance in Parkinson's disease: Critique and recommendations. Mov Disord. 2016;31(9):1342-1355.

21. Hausdorff JM. Gait dynamics, fractals and falls: finding meaning in the stride-to-stride fluctuations of human walking. Hum Mov Sci. 2007; 26(4):555-589.

22. Bohannon RW, Andrews AW, Thomas MW. Walking speed: reference values and correlates for older adults. J Orthop Sports Phys Ther. 1996; 24(2):86-90.

23. Tomlinson CL, Patel S, Meek C, et al. Physiotherapy intervention in Parkinson's disease: systematic review and meta-analysis. BMJ. 2012; 345:e5004.

24. Lord S, Galna B, Rochester L. Moving forward on gaitmeasurement: toward a more refined approach. Mov Disord. 2013;28(11):1534-1543.

25. Hausdorff JM. Gait dynamics in Parkinson's disease: common and distinct behavior among stride length, gait variability, and fractal-like scaling. Chaos. 2009;19(2):026113.

26. Hausdorff JM. Gait variability: methods, modeling and meaning. J Neuroeng Rehabil. 2005;2:19.

27. Lord S, Chastin SF, McInnes L, Little L, Briggs P, Rochester L. Exploring patterns of daily physical and sedentary behavior in communitydwelling older adults. Age Aging. 2011;40(2):205-210.

28. Brach JS, Perera S, Studenski S, Katz M, Hall C, Verghese J. Meaningful change in measures of gait variability in older adults. Gait Posture. 2010;31(2):175-179.

29. Montero-Odasso M, Wells JL, Borrie MJ, Speechley M. Can cognitive enhancers reduce the risk of falls in older people with mild cognitive impairment? A protocol for a randomized controlled double blind trial. BMC Neurol. 2009;9:42.

30. Segev-Jacubovski O, Herman T, Yogev-Seligmann G, Mirelman A, Giladi N, Hausdorff JM. The interplay between gait, falls and cognition: can cognitive therapy reduce fall risk? Expert Rev Neurother. 2011; 11(7):1057-1075

31. Amboni M, Barone P, Hausdorff JM. Cognitive contributions to gait and falls: evidence and implications. Mov Disord. 2013;28(11):1520-1533.

32. Liu-Ambrose T, Nagamatsu LS, Graf P, Beattie BL, Ashe MC, Handy TC. Resistance training and executive functions: a 12-month randomized controlled trial. Arch Intern Med. 2010;170(2):170-178.

33. Verghese J, Mahoney J, Ambrose AF, Wang C, Holtzer R. Effect of cognitive remediation on gait in sedentary seniors. $J$ Gerontol A Biol Sci Med Sci. 2010;65(12):1338-1343.

34. Holtzer R, Wang C, Verghese J. The relationship between attention and gait in aging: facts and fallacies. Motor Control. 2012;16(1):64-80.

35. Abellan van Kan G, Rolland Y, Andrieu S, et al. Gait speed at usual pace as a predictor of adverse outcomes in community-dwelling older people an International Academy on Nutrition and Aging (IANA) Task Force. J Nutr Health Aging. 2009;13(10):881-889.

36. Lim LI, van Wegen EE, de Goede CJ, et al. Measuring gait and gaitrelated activities in Parkinson's patients own home environment: a reliability, responsiveness and feasibility study. Parkinsonism Relat Disord. 2005;11(1):19-24.

37. Steffen T, Seney M. Test-retest reliability and minimal detectable change on balance and ambulation tests, the 36-item short-form health survey, and the unified Parkinson disease rating scale in people with parkinsonism. Phys Ther. 2008;88(6):733-746.

38. Galna B, Lord S, Rochester L. Is gait variability reliable in older adults and Parkinson's disease? Towards an optimal testing protocol. Gait Posture. 2013;37(4):580-585.

39. Hollman JH, Childs KB, McNeil ML, Mueller AC, Quilter CM, Youdas JW. Number of strides required for reliable measurements of pace, rhythm and variability parameters of gait during normal and dual task walking in older individuals. Gait Posture. 2010;32(1):23-28.

40. Paterson KL, Lythgo ND, Hill KD. Gait variability in younger and older adult women is altered by overground walking protocol. Age Aging. 2009;38(6):745-748. 
41. Mehrholz J, Kugler J, Storch A, Pohl M, Elsner B, Hirsch K. Treadmill training for patients with Parkinson's disease. Cochrane Database Syst Rev. 2015;(8):CD007830.

42. Bello O, Sanchez JA, Lopez-Alonso V, et al. The effects of treadmill or overground walking training program on gait in Parkinson's disease. Gait Posture. 2013;38(4):590-595.

43. Verghese J, Holtzer R, Lipton RB, Wang C. Mobility stress test approach to predicting frailty, disability, and mortality in high-functioning older adults. J Am Geriatr Soc. 2012;60(10):1901-1905.

44. Stegemoller EL, Wilson JP, Hazamy A, et al. Associations between cognitive and gait performance during single- and dual-task walking in people with Parkinson disease. Phys Ther. 2014;94(6):757-766.

45. Smulders K, Esselink RA, Weiss A, Kessels RP, Geurts AC, Bloem BR. Assessment of dual tasking has no clinical value for fall prediction in Parkinson's disease. J Neurol. 2012;259(9):1840-1847.

46. Pieruccini-Faria F, Jones JA, Almeida QJ. Insight into dopaminedependent planning deficits in Parkinson's disease: a sharing of cognitive \& sensory resources. Neuroscience. 2016;318:219-229.

47. Yiou E, Artico R, Teyssedre CA, Labaune O, Fourcade P. Anticipatory postural control of stability during gait initiation over obstacles of different height and distance made under reaction-time and self-initiated instructions. Front Hum Neurosci. 2016;10:449.

48. Rogers MW, Hilliard MJ, Martinez KM, Zhang Y, Simuni T, Mille ML. Perturbations of ground support alter posture and locomotion coupling during step initiation in Parkinson's disease. Exp Brain Res. 2011; 208(4):557-567.
49. Burleigh-Jacobs A, Horak FB, Nutt JG, Obeso JA. Step initiation in Parkinson's disease: influence of levodopa and external sensory triggers. Mov Disord. 1997;12(2):206-215.

50. Chong RK, Horak FB, Woollacott MH. Parkinson's disease impairs the ability to change set quickly. J Neurol Sci. 2000;175(1):57-70.

51. Jacobs JV, Horak FB. Abnormal proprioceptive-motor integration contributes to hypometric postural responses of subjects with Parkinson's disease. Neuroscience. 2006;141(2):999-1009.

52. Chang YT, Huang CF, Chang JH. The effect of Tai Chi Chuan on obstacle crossing strategy in older adults. Res Sports Med. 2015;23(3) 315-329.

53. Dadashi F, Mariani B, Rochat S, Bula CJ, Santos-Eggimann B, Aminian K. Gait and foot clearance parameters obtained using shoeworn inertial sensors in a large-population sample of older adults. Sensors (Basel). 2013;14(1):443-457.

54. Bloem BR, de Vries NM, Ebersbach G. Nonpharmacological treatments for patients with Parkinson's disease. Mov Disord. 2015;30(11) 1504-1520.

55. Nakano W, Fukaya T, Kanai Y, Akizuki K, Ohashi Y. Effects of temporal constraints on medio-lateral stability when negotiating obstacles. Gait Posture. 2015;42(2):158-164.

56. Ida H, Mohapatra S, Aruin A. Control of vertical posture while elevating one foot to avoid a real or virtual obstacle. Exp Brain Res. 2017; 235(6):1677-1687.
Clinical Interventions in Aging

\section{Publish your work in this journal}

Clinical Interventions in Aging is an international, peer-reviewed journal focusing on evidence-based reports on the value or lack thereof of treatments intended to prevent or delay the onset of maladaptive correlates of aging in human beings. This journal is indexed on PubMed Central, MedLine,

\section{Dovepress}

CAS, Scopus and the Elsevier Bibliographic databases. The manuscript management system is completely online and includes a very quick and fair peer-review system, which is all easy to use. Visit http://www.dovepress. com/testimonials.php to read real quotes from published authors. 